\title{
Necessary Conditions for Convergence Rates of Regularizations of Optimal Control Problems
}

\author{
Daniel Wachsmuth and Gerd Wachsmuth \\ Johann Radon Institute for Computational and Applied Mathematics (RICAM), \\ Austrian Academy of Sciences, Altenbergerstraße 69, A-4040 Linz, Austria, \\ Chemnitz University of Technology, Department of Mathematics, D-09107 Chemnitz, \\ Germany
}

\begin{abstract}
We investigate the Tikhonov regularization of control constrained optimal control problems. We use a specialized source condition in combination with a condition on the active sets. In the case of high convergence rates, these conditions are necessary and sufficient.
\end{abstract}

Keywords: optimal control problem, inequality constraints, Tikhonov regularization, source condition.

\section{Introduction}

In this article, we investigate regularization schemes for the following class of optimization problems:

$$
\begin{array}{ll}
\text { Minimize } & \frac{1}{2}\|\mathcal{S} u-z\|_{Y}^{2}+\beta\|u\|_{L^{1}(\Omega)} \\
\text { such that } & u \in L^{2}(\Omega) \text { and } u_{a} \leq u \leq u_{b} \text { a.e. on } \Omega .
\end{array}
$$

Here, $\Omega$ is a measurable subset of $\mathbb{R}^{n}, n \geq 1, Y$ is a Hilbert space, $\mathcal{S}: L^{2}(\Omega) \rightarrow Y$ a bounded linear operator, and the function $z \in Y$ is given. The parameter $\beta$ is assumed to be non-negative. The control constraints $u_{a}, u_{b} \in L^{\infty}(\Omega)$ satisfy $u_{a} \leq 0 \leq u_{b}$.

This model problem can be interpreted as an optimal control problem as well as an inverse problem. In the point of view of inverse problems, the unknown $u$ has to be constructed in order to reproduce given measurements $z$. The inequality constraints on $u$ reflect certain a-priori knowledge about the solution $u^{\dagger}$ of the linear ill-posed equation $\mathcal{S} u=z$. If the problem at hand is seen as an optimal control problem, then $u$ is the control, $\mathcal{S} u$ the state of the system, which has to be close to a desired state $z$, the inequality constraints restrict the feasible set and may hinder the state $\mathcal{S} u$ to reach the target $z$. If the parameter $\beta$ is positive, then the resulting optimal control will be sparse, that is, its support is a possibly small subset of $\Omega$.

The resulting optimization problem $(\mathrm{P})$ is nevertheless ill-posed if $\mathcal{S}$ is not continuously invertible. Due to the control constraints, problem $(\mathrm{P})$ still possesses a solution, which is even unique if $\mathcal{S}$ is injective. However, the solution 
may be unstable with respect to perturbations in the problem data, for instance in the given state $z$. Here small perturbations due to measurement errors may lead to large changes in the solution. Consequently, any numerical approximation of $(\mathrm{P})$ is challenging to solve and numerical approximations of solutions may converge arbitrarily slow. Let us note, that a positive value of $\beta$ does not make the problem well-posed. This is due to the fact, that $L^{1}(\Omega)$ is not a dual space and hence bounded sets in $L^{1}(\Omega)$ are not compact w.r.t. the weak(-star) topology, see also the discussions in $[7,8]$.

In order to overcome this difficulty, we apply common ideas from inverse problem theory. We will study a regularization of the type

$$
\begin{array}{ll}
\text { Minimize } & \frac{1}{2}\|\mathcal{S} u-z\|_{Y}^{2}+\beta\|u\|_{L^{1}(\Omega)}+\frac{\alpha}{2}\|u\|_{L^{2}(\Omega)}^{2} \\
\text { such that } & u \in L^{2}(\Omega) \text { and } u_{a} \leq u \leq u_{b} \text { a.e. on } \Omega,
\end{array}
$$

where $\alpha>0$ is given. Clearly, the problems $\left(\mathrm{P}_{\alpha}\right)$ are uniquely solvable for $\alpha>$ 0 . Now, the question arises, whether their solutions $u_{\alpha}$ converge (weakly or strongly) to a solution $u_{0}$ of (P) for $\alpha \rightarrow 0$. Moreover, in the case of convergence, one is interested in proving convergence rates of $\left\|u_{\alpha}-u_{0}\right\|_{L^{2}(\Omega)}$ and $\left\|\mathcal{S} u_{\alpha}-\mathcal{S} u_{0}\right\|_{Y}$ under suitable assumptions.

In this work, we will prove necessary conditions for convergence rates. In some parts, the necessary conditions are similar to sufficient conditions found in earlier works [7,8]. Moreover, the result of Theorem 3 leads to a weakened sufficient condition for convergence rates.

\subsection{Standing Assumptions and Notation}

Let us fix the standing assumptions on the problem $(\mathrm{P})$. We assume that $\mathcal{S}$ : $L^{2}(\Omega) \rightarrow Y$ is linear and continuous. In many applications this operator $\mathcal{S}$ is compact. Furthermore, we assume that the Hilbert space adjoint operator $\mathcal{S}^{\star}$ maps into $L^{\infty}(\Omega)$, i.e., $\mathcal{S}^{\star} \in \mathcal{L}\left(Y, L^{\infty}(\Omega)\right)$. These assumptions imply that the range of $\mathcal{S}$ is closed in $Y$ if and only if the range of $\mathcal{S}$ is finite-dimensional, see [8, Prop. 2.1]. Hence, up to trivial cases, (P) is ill-posed. A typical example for $\mathcal{S}$ is the solution operator of the Poisson problem with homogeneous Dirichlet boundary conditions.

The set of feasible functions $u$ is given by

$$
U_{a d}:=\left\{u \in L^{2}(\Omega): u_{a} \leq u \leq u_{b} \text { a.e. on } \Omega\right\} .
$$

The problem $\left(\mathrm{P}_{\alpha}\right)$ is uniquely solvable for $\alpha>0$. We will denote its solution by $u_{\alpha}$, with the corresponding state $y_{\alpha}:=\mathcal{S} u_{\alpha}$ and adjoint state $p_{\alpha}:=\mathcal{S}^{\star}\left(z-y_{\alpha}\right)$. There is a unique solution of $(\mathrm{P})$ with minimal $L^{2}(\Omega)$ norm, see [8, Thm. 2.3, Lem. 2.7]. This solution and the associated state and adjoint state will be denoted by $u_{0}, y_{0}$ and $p_{0}$, respectively. Note that the weak convergence $u_{\alpha} \rightarrow u^{\star}$ in $L^{2}(\Omega)$, where $u^{\star}$ is a solution of $(\mathrm{P})$ already implies $u^{\star}=u_{0}$, see [8, Rem. 3.3]. 


\subsection{Optimality Conditions}

As both problems $(\mathrm{P})$ and $\left(\mathrm{P}_{\alpha}\right)$ are convex, their solutions can be characterized by the following necessary and sufficient optimality conditions:

Theorem 1 ([7, Lemma 2.2]). Let $\alpha \geq 0$ be given, and let $u_{\alpha}$ be a solution of $\left(\mathrm{P}_{\alpha}\right)$ (or $(\mathrm{P})$ in the case $\left.\alpha=0\right)$.

Then, there exists a subgradient $\lambda_{\alpha} \in \partial\left\|u_{\alpha}\right\|_{L^{1}(\Omega)}$, such that the variational inequality

$$
\left(\alpha u_{\alpha}-p_{\alpha}+\beta \lambda_{\alpha}, u-u_{\alpha}\right) \geq 0 \quad \forall u \in U_{a d},
$$

is satisfied, where $p_{\alpha}=\mathcal{S}^{\star}\left(z-\mathcal{S} u_{\alpha}\right)$ is the associated adjoint state.

Here, $(\cdot, \cdot)$ refers to the scalar product in $L^{2}(\Omega)$.

Standard arguments (see [6, Section 2.8]) lead to a pointwise a.e. interpretation of the variational inequality, which in turn implies the following relation between $u_{\alpha}$ and $p_{\alpha}$ in the case $\alpha>0$ :

$$
u_{\alpha}(x)=\left\{\begin{array}{lll}
u_{a}(x) & \text { if } p_{\alpha}(x)<\alpha u_{a}(x)-\beta & \\
\frac{1}{\alpha}\left(p_{\alpha}(x)+\beta\right) & \text { if } \alpha u_{a}(x)-\beta \leq p_{\alpha}(x) \leq-\beta & \\
0 & \text { if }\left|p_{\alpha}(x)\right|<\beta & \text { a.e. on } \Omega . \\
\frac{1}{\alpha}\left(p_{\alpha}(x)-\beta\right) & \text { if } \beta \leq p_{\alpha}(x) \leq \alpha u_{b}(x)+\beta & \\
u_{b}(x) & \text { if } \alpha u_{b}(x)+\beta<p_{\alpha}(x) &
\end{array}\right.
$$

In the case $\alpha=0$, we have

$$
u_{0}(x)\left\{\begin{array}{ll}
=u_{a}(x) & \text { if } p_{0}(x)<-\beta \\
\in\left[u_{a}(x), 0\right] & \text { if } p_{0}(x)=-\beta \\
=0 & \text { if }\left|p_{0}(x)\right|<\beta \\
\in\left[0, u_{b}(x)\right] & \text { if } p_{0}(x)=\beta \\
=u_{b}(x) & \text { if } \beta<p_{0}(x)
\end{array} \text { a.e. on } \Omega .\right.
$$

Note that if $\beta=0$, one obtains $u_{0}(x) \in\left[u_{a}(x), u_{b}(x)\right]$ where $p_{0}(x)=0$ in (3). This implies that $u_{0}(x)$ is uniquely determined by $p_{0}(x)$ on the set, where it holds $\left|p_{0}(x)\right| \neq \beta$.

\section{Sufficient Conditions for Convergence Rates}

Let us first recall the sufficient conditions for convergence rates as obtained in [8]. We will work with the following assumption. There we denote by $\operatorname{proj}_{[a, b]}(v)$ the projection of the real number $v$ onto the interval $[a, b]$.

Assumption 2. Let $u_{0}$ be a solution of $(\mathrm{P})$. Let us assume that there exist a measurable set $I \subset \Omega$, a function $w \in Y$, and positive constants $\kappa, c$ such that it holds: 
1. (source condition) $I \supset\left\{x \in \Omega:\left|p_{0}(x)\right|=\beta\right\}$, and for almost all $x \in I$

$$
u_{0}(x)= \begin{cases}\operatorname{proj}_{\left[u_{a}(x), 0\right]}\left(\left(\mathcal{S}^{\star} w\right)(x)\right) & \text { if } \beta>0, p_{0}(x) \leq-\frac{\beta}{2}, \\ \operatorname{proj}_{\left[0, u_{b}(x)\right]}\left(\left(\mathcal{S}^{\star} w\right)(x)\right) & \text { if } \beta>0, p_{0}(x) \geq \frac{\beta}{2}, \\ \operatorname{proj}_{\left[u_{a}(x), u_{b}(x)\right]}\left(\left(\mathcal{S}^{\star} w\right)(x)\right) & \text { if } \beta=0 .\end{cases}
$$

2. (structure of active set) $A=\Omega \backslash I$ and for all $\epsilon>0$

$$
\begin{aligned}
& \operatorname{meas}\left(\left\{x \in A: 0<|| p_{0}(x)|-\beta|<\epsilon\right\}\right) \leq c \epsilon^{\kappa} \quad \text { if } w \neq 0, \\
& \operatorname{meas}\left(\left\{x \in A: 0<\left|p_{0}(x)\right|-\beta<\epsilon\right\}\right) \leq c \epsilon^{\kappa} \quad \text { if } w=0 .
\end{aligned}
$$

Some remarks are in order. The first part of the assumption is analogous to source conditions in inverse problems: we assume that on the set $I \subset \Omega$ the solution $u_{0}$ is the restriction to $I$ of a certain pointwise projection of an element in the range of $\mathcal{S}^{\star}$. This part of the condition is different from other conditions in the literature: in our earlier work [8] we used the assumption $u_{0}(x)=\operatorname{proj}_{\left[u_{a}(x), u_{b}(x)\right]}\left(\left(\mathcal{S}^{\star} w\right)(x)\right)$ on $I$. However, in the light of the derivation of necessary conditions it turns out that such a condition can be weakened without losing anything with respect to convergence rates. In works on inverse problems [3,5], the source condition $u_{0}=\operatorname{proj}_{U_{a d}}\left(\mathcal{S}^{\star} w\right)$ is used, which is retained as the special case $I=\Omega$ in Assumption 2 .

The assumption (5) (without the second alternative) on the active sets was already employed to obtain regularization error estimates $[7,8]$, error estimates for finite-element discretizations of $(\mathrm{P})$ [2], as well as stability results of bang-bang controls [4]. Note that in the case $\beta=0$, both conditions in (5) are equivalent. However, if $\beta>0$ and $w=0$ (in particular, if $I$ has measure zero), the second alternative provides a weaker condition than the first one. Hence, condition (5) is weaker than the condition used in our earlier work [8].

Theorem 3. Let Assumption 2 be satisfied.

Let $d$ be defined as

$$
d= \begin{cases}\frac{1}{2-\kappa} & \text { if } \kappa \leq 1, \\ 1 & \text { if } \kappa>1 \text { and } w \neq 0, \\ \frac{\kappa+1}{2} & \text { if } \kappa>1 \text { and } w=0 .\end{cases}
$$

Then there is $\alpha_{\max }>0$ and a constant $c>0$, such that

$$
\begin{aligned}
\left\|y_{0}-y_{\alpha}\right\|_{Y} & \leq c \alpha^{d} \\
\left\|p_{0}-p_{\alpha}\right\|_{L^{\infty}(\Omega)} & \leq c \alpha^{d} \\
\left\|u_{0}-u_{\alpha}\right\|_{L^{2}(\Omega)} & \leq c \alpha^{d-1 / 2}
\end{aligned}
$$

holds for all $\alpha \in\left(0, \alpha_{\max }\right]$.

Under the assumptions of the theorem, one can prove also convergence rates for $\left\|u_{\alpha}-u_{0}\right\|_{L^{1}(A)}[8]$. 
Proof. The proof is analogous to the proof of [8, Thm. 3.14]. We have to take into account the modification of the source condition (4) in the case $\beta>0$ and the modification of (5) in the case $w=0$. By [8, Lemma 2.12], we have

$$
\left\|y_{0}-y_{\alpha}\right\|_{Y}^{2}+\alpha\left\|u_{0}-u_{\alpha}\right\|_{L^{2}(\Omega)}^{2} \leq \alpha\left(u_{0}, u_{0}-u_{\alpha}\right) .
$$

Since $U_{a d}$ is bounded, we obtain $\left\|p_{0}-p_{\alpha}\right\|_{L^{\infty}(\Omega)} \leq c \alpha^{1 / 2}$ for some $c>0$ independent of $\alpha$.

Let now $\alpha$ be small enough such that $\left\|p_{0}-p_{\alpha}\right\|_{L^{\infty}(\Omega)}<\beta / 2$. This implies that $p_{0}$ and $p_{\alpha}$ have the same sign on the set $\left\{x \in I:\left|p_{0}(x)\right| \geq \beta / 2\right\}$. Consequently, $u_{0}$ and $u_{\alpha}$ have the same sign on this set, too. Moreover, on the set $\{x \in I$ : $\left.\left|p_{0}(x)\right|<\beta / 2\right\}$ it holds $\left|p_{\alpha}\right|<\beta$, and hence $u_{\alpha}=0=u_{0}$ on this set. This yields

$$
\left(\chi_{I} u_{0}, u_{0}-u_{\alpha}\right) \leq\left(\chi_{I} \mathcal{S}^{\star} w, u_{0}-u_{\alpha}\right)
$$

for $\alpha>0$ small enough. Note that in case of $w=0$, the right-hand side in the previous estimate vanishes and it remains to estimate $\left(\chi_{A} u_{0}, u_{0}-u_{\alpha}\right)$. Taking into account that $u_{0}(x)=0$ whenever $\left|p_{0}(x)\right|<\beta$, the weakend estimate (5) is sufficient in this case. Arguing as in the proof of [8, Thm. 3.14] proves the claim.

\section{Necessary Conditions for Convergence Rates}

\subsection{Necessity of the Source Condition (4)}

Theorem 4. Let us suppose that $\left\|y_{\alpha}-y_{0}\right\|_{Y}=O(\alpha)$ with $y_{0}=\mathcal{S} u_{0}$. Then there exists $w \in Y$ such that

$$
u_{0}(x)= \begin{cases}\operatorname{proj}_{\left[u_{a}(x), 0\right]}\left(\left(\mathcal{S}^{\star} w\right)(x)\right) & \text { if } \beta>0, p_{0}(x)=-\beta, \\ \operatorname{proj}_{\left[0, u_{b}(x)\right]}\left(\left(\mathcal{S}^{\star} w\right)(x)\right) & \text { if } \beta>0, p_{0}(x)=+\beta, \\ \operatorname{proj}_{\left[u_{a}(x), u_{b}(x)\right]}\left(\left(\mathcal{S}^{\star} w\right)(x)\right) & \text { if } \beta=0, p_{0}(x)=0 .\end{cases}
$$

If moreover $\left\|y_{\alpha}-y_{0}\right\|_{Y}=o(\alpha)$, then $u_{0}=0$ on $\left\{x \in \Omega:\left|p_{0}(x)\right|=\beta\right\}$, i.e. $w=0$.

This result shows that the source condition (4) is necessary on the set $\{x \in \Omega$ : $\left.\left|p_{0}(x)\right|=\beta\right\}$.

Proof. Let us prove the claim in the case $\beta>0$. The result in the case $\beta=0$ can be proved with obvious modifications. Let us take a test function $u \in U_{a d}$ defined as

$$
u(x) \begin{cases}=u_{a}(x) & \text { if } p_{0}(x)<-\beta, \\ \in\left[u_{a}(x), 0\right] & \text { if } p_{0}(x)=-\beta, \\ =0 & \text { if }\left|p_{0}(x)\right|<\beta, \\ \in\left[0, u_{b}(x)\right] & \text { if } p_{0}(x)=\beta, \\ =u_{b}(x) & \text { if } p_{0}(x)>\beta .\end{cases}
$$


Due to the relation

$$
\lambda_{0}=\operatorname{proj}_{[-1,1]}\left(\frac{1}{\beta} p_{0}\right)
$$

which is a consequence of the necessary optimality condition, see [1] for a proof, we obtain $\lambda_{0}= \pm 1$ where $p_{0}= \pm \beta$. Hence it holds

$$
\left(-p_{0}, u-u_{0}\right)=\left(-\beta \lambda_{0}, u-u_{0}\right)=\beta\left\|u_{0}\right\|_{L^{1}(\Omega)}-\beta\|u\|_{L^{1}(\Omega)}
$$

for $u$ as above.

Since $\lambda_{0} \in \partial\left\|u_{0}\right\|_{L^{1}(\Omega)}$, we obtain

$$
\left(\lambda_{0}, u_{\alpha}-u_{0}\right) \leq\left\|u_{\alpha}\right\|_{L^{1}(\Omega)}-\left\|u_{0}\right\|_{L^{1}(\Omega)} .
$$

Using the optimality of $u_{\alpha}$ and the relation $-p_{\alpha}=-p_{0}+\mathcal{S}^{\star} \mathcal{S}\left(u_{\alpha}-u_{0}\right)$ we get

$$
\left(-p_{0}+\mathcal{S}^{\star} \mathcal{S}\left(u_{\alpha}-u_{0}\right)+\alpha u_{\alpha}, u-u_{\alpha}\right)+\beta\|u\|_{L^{1}(\Omega)}-\beta\left\|u_{\alpha}\right\|_{L^{1}(\Omega)} \geq 0 .
$$

Adding $\left(-p_{0}+\beta \lambda_{0}, u_{\alpha}-u_{0}\right) \geq 0$ to the left-hand side yields

$$
\begin{aligned}
&\left(\mathcal{S}^{\star} \mathcal{S}\left(u_{\alpha}-u_{0}\right)+\alpha u_{\alpha}, u-u_{\alpha}\right)+\left(-p_{0},\right.\left.u-u_{0}\right)+\left(\beta \lambda_{0}, u_{\alpha}-u_{0}\right) \\
&+\beta\|u\|_{L^{1}(\Omega)}-\beta\left\|u_{\alpha}\right\|_{L^{1}(\Omega)} \geq 0 .
\end{aligned}
$$

Using (8) and (9) we obtain

$$
\left(\mathcal{S}^{\star} \mathcal{S}\left(u_{\alpha}-u_{0}\right)+\alpha u_{\alpha}, u-u_{\alpha}\right) \geq 0 .
$$

Due to the assumptions of the theorem, the functions $\frac{1}{\alpha}\left(\mathcal{S}\left(u_{\alpha}-u_{0}\right)\right)=\frac{1}{\alpha}\left(y_{\alpha}-y_{0}\right)$ are uniformly bounded for $\alpha \searrow 0$. As a consequence, $\alpha \searrow 0$ implies

$$
\left(\mathcal{S}^{\star} \dot{y}_{0}+u_{0}, u-u_{0}\right) \geq 0
$$

for any weak subsequential limit $\dot{y}_{0}$ of $\frac{1}{\alpha}\left(y_{\alpha}-y_{0}\right)$. Due to the construction of the test function $u$, we obtain

$$
u_{0}= \begin{cases}\operatorname{proj}_{\left[u_{a}, 0\right]}\left(\mathcal{S}^{\star} \dot{y}_{0}\right) & \text { where } p_{0}=-\beta, \\ \operatorname{proj}_{\left[0, u_{b}\right]}\left(\mathcal{S}^{\star} \dot{y}_{0}\right) & \text { where } p_{0}=+\beta .\end{cases}
$$

If $\left\|y_{\alpha}-y_{0}\right\|_{Y}=o(\alpha)$ then $\frac{1}{\alpha}\left(y_{\alpha}-y_{0}\right) \rightarrow 0$ strongly in $Y$ for $\alpha \rightarrow 0$, hence $\dot{y}_{0}=0$, and $u_{0}=0$ on the set $\left\{\left|p_{0}\right|=\beta\right\}$.

As can be seen from the proof, the element that realizes the source condition can be interpreted as the (weak) directional derivate of $\alpha \mapsto y_{\alpha}$ at $\alpha=0$.

The result of the theorem resembles known results of necessity of the source condition in linear inverse problems, see e.g. [3,5]. 


\subsection{Necessity of the Condition (5) on the Active Set}

In this section, we want to prove the necessity of (5) in the case of high convergence rates $d>1$. In this case, we have $w=0$, see Theorem 4 . It remains to show that the second condition in (5) is necessary to obtain convergence rates $d>1$. Hence, we derive a bound on

$$
\mu(\epsilon):=\left|\left\{x \in \Omega: 0<\left|p_{0}(x)\right|-\beta<\epsilon\right\}\right|,
$$

which is the measure of a subset of

$$
A:=\left\{x \in \Omega: \beta<\left|p_{0}(x)\right|\right\} .
$$

For $\alpha>0$ let $\tilde{u}_{\alpha}$ denote the unique solution of

$$
\min _{u \in U_{a d}}-\left(u, p_{0}\right)+\frac{\alpha}{2}\|u\|_{L^{2}(\Omega)}^{2}+\beta\|u\|_{L^{1}(\Omega)} .
$$

Analogous to (2), we have the representation

$$
\tilde{u}_{\alpha}(x)=\left\{\begin{array}{lll}
u_{a}(x) & \text { if } p_{0}(x)<\alpha u_{a}(x)-\beta & \\
\frac{1}{\alpha}\left(p_{0}(x)+\beta\right) & \text { if } \alpha u_{a}(x)-\beta \leq p_{0}(x) \leq-\beta & \\
0 & \text { if }\left|p_{0}(x)\right|<\beta & \text { a.e. on } \Omega . \\
\frac{1}{\alpha}\left(p_{0}(x)-\beta\right) & \text { if } \beta \leq p_{0}(x) \leq \alpha u_{b}(x)+\beta & \\
u_{b}(x) & \text { if } \alpha u_{b}(x)+\beta<p_{0}(x) &
\end{array}\right.
$$

Let us first prove a relation between the convergence rates of $\left\|u_{0}-\tilde{u}_{\alpha}\right\|_{L^{2}(A)}$ and $\mu(\epsilon)$ for $\alpha \rightarrow 0$ and $\epsilon \rightarrow 0$, respectively.

Lemma 5. Let us assume that there is $\sigma>0$ such that $u_{a} \leq-\sigma<0<\sigma \leq u_{b}$ a.e. on $\Omega$. Then it holds: If $\left\|u_{0}-\tilde{u}_{\alpha}\right\|_{L^{2}(A)}=O\left(\alpha^{d}\right), d>0$, for $\alpha \rightarrow 0$, then $\mu(\epsilon)=O\left(\epsilon^{2 d}\right)$ for $\epsilon \rightarrow 0$.

Proof. Due to the pointwise representations of $\tilde{u}_{\alpha}$ and $u_{0}$ in (10) and (3), respectively, it holds

$$
\begin{aligned}
\left\|u_{0}-\tilde{u}_{\alpha}\right\|_{L^{2}(A)}^{2}=\int_{\left\{\beta<p_{0}<\alpha u_{b}+\beta\right\}}\left(u_{b}-\alpha^{-1}\left(p_{0}-\beta\right)\right)^{2} & \\
& +\int_{\left\{\alpha u_{a}-\beta<p_{0}<-\beta\right\}}\left(u_{a}-\alpha^{-1}\left(p_{0}+\beta\right)\right)^{2} .
\end{aligned}
$$

Due to the assumption on the control constraints we have

$$
\begin{aligned}
\int_{\left\{\beta<p_{0}<\alpha u_{b}+\beta\right\}}\left(u_{b}-\alpha^{-1}\left(p_{0}-\beta\right)\right)^{2} & \geq \int_{\left\{\beta<p_{0}<\alpha \sigma / 2+\beta\right\}}\left(u_{b}-\alpha^{-1}\left(p_{0}-\beta\right)\right)^{2} \\
& \geq \int_{\left\{\beta<p_{0}<\alpha \sigma / 2+\beta\right\}}(\sigma / 2)^{2} \\
& \geq(\sigma / 2)^{2}\left|\left\{x \in \Omega: 0<p_{0}(x)-\beta<\alpha \sigma / 2\right\}\right| .
\end{aligned}
$$


Similarly, we obtain

$\int_{\left\{\alpha u_{a}-\beta<p_{0}<-\beta\right\}}\left(u_{a}-\alpha^{-1}\left(p_{0}+\beta\right)\right)^{2} \geq(\sigma / 2)^{2}\left|\left\{x \in \Omega: 0<-p_{0}(x)-\beta<\alpha \sigma / 2\right\}\right|$.

This implies

$$
\left\|u_{0}-\tilde{u}_{\alpha}\right\|_{L^{2}(A)}^{2} \geq(\sigma / 2)^{2} \mu(\alpha \sigma / 2) .
$$

Hence if $\left\|u_{0}-\tilde{u}_{\alpha}\right\|_{L^{2}(A)}=O\left(\alpha^{d}\right)$ holds, then

$$
\mu(\alpha \sigma / 2) \leq O\left(\alpha^{2 d}\right),
$$

for $\alpha \rightarrow 0$, which proves the claim.

Using the same arguments, we can prove the following result.

Corollary 6. Let the requirements of Theorem 5 be satisfied. Let $p \in[1, \infty)$ be given. Then it holds

$$
\left(\frac{\sigma}{2}\right)^{p} \mu\left(\frac{\sigma}{2} \alpha\right) \leq\left\|u_{0}-\tilde{u}_{\alpha}\right\|_{L^{p}(A)}^{p} \leq M^{p} \mu(M \alpha)
$$

with $M=\max \left(\left\|u_{a}\right\|_{L^{\infty}(\Omega)},\left\|u_{b}\right\|_{L^{\infty}(\Omega)}\right)$.

Lemma 7. Let $\tilde{u}_{\alpha}$ be defined as above. Then it holds

$$
\alpha\left\|\tilde{u}_{\alpha}-u_{\alpha}\right\|_{L^{2}(\Omega)}^{2}+\left\|y_{0}-y_{\alpha}\right\|_{Y}^{2} \leq\left(p_{0}-p_{\alpha}, \tilde{u}_{\alpha}-u_{0}\right) .
$$

Proof. Since $u_{\alpha}$ and $\tilde{u}_{\alpha}$ solve $\left(\mathrm{P}_{\alpha}\right)$ and $\left(\mathrm{P}_{\alpha}^{\mathrm{aux}}\right)$, respectively, we have

$$
\begin{gathered}
\left(\alpha u_{\alpha}-p_{\alpha}+\beta \lambda_{\alpha}, \tilde{u}_{\alpha}-u_{\alpha}\right) \geq 0, \\
\left(\alpha \tilde{u}_{\alpha}-p_{0}+\beta \tilde{\lambda}_{\alpha}, u_{\alpha}-\tilde{u}_{\alpha}\right) \geq 0,
\end{gathered}
$$

with some $\tilde{\lambda}_{\alpha} \in \partial\left\|\tilde{u}_{\alpha}\right\|_{L^{1}(\Omega)}$. Due to the monotonicity of the subdifferential we have $\left(\lambda_{\alpha}-\tilde{\lambda}_{\alpha}, u_{\alpha}-\tilde{u}_{\alpha}\right) \geq 0$. This gives

$$
\alpha\left\|\tilde{u}_{\alpha}-u_{\alpha}\right\|_{L^{2}(\Omega)}^{2} \leq\left(p_{0}-p_{\alpha}, \tilde{u}_{\alpha}-u_{\alpha}\right) .
$$

The identity

$$
\begin{aligned}
\left(p_{0}-p_{\alpha}, \tilde{u}_{\alpha}-u_{\alpha}\right) & =\left(p_{0}-p_{\alpha}, \tilde{u}_{\alpha}-u_{0}+u_{0}-u_{\alpha}\right) \\
& =\left(p_{0}-p_{\alpha}, \tilde{u}_{\alpha}-u_{0}\right)-\left\|y_{0}-y_{\alpha}\right\|_{Y}^{2}
\end{aligned}
$$

finishes the proof.

Theorem 8. Let us assume that there is $\sigma>0$ such that $u_{a} \leq-\sigma<0<\sigma \leq u_{b}$ a.e. on $\Omega$. The we have the following implication: If

$$
\left\|u_{0}-u_{\alpha}\right\|_{L^{2}(\Omega)}=O\left(\alpha^{d-1 / 2}\right), \quad\left\|y_{0}-y_{\alpha}\right\|_{Y}=O\left(\alpha^{d}\right) \text { for } \alpha \rightarrow 0
$$

holds with $d>1$, then it follows

$$
\mu(\epsilon) \leq O\left(\epsilon^{2 d-1}\right) \text { for } \epsilon \rightarrow 0 .
$$


Proof. Let us begin with

$$
\begin{aligned}
\left\|u_{0}-\tilde{u}_{\alpha}\right\|_{L^{2}(\Omega)}^{2} & \leq 2\left(\left\|u_{0}-u_{\alpha}\right\|_{L^{2}(\Omega)}^{2}+\left\|u_{\alpha}-\tilde{u}_{\alpha}\right\|_{L^{2}(\Omega)}^{2}\right) \\
& \leq O\left(\alpha^{2 d-1}\right)+\alpha^{-1}\left(p_{0}-p_{\alpha}, \tilde{u}_{\alpha}-u_{0}\right) \\
& \leq O\left(\alpha^{2 d-1}\right)+O\left(\alpha^{d-1}\right)\left\|u_{0}-\tilde{u}_{\alpha}\right\|_{L^{2}(\Omega)},
\end{aligned}
$$

which gives $\left\|u_{0}-\tilde{u}_{\alpha}\right\|_{L^{2}(\Omega)}=O\left(\alpha^{d-1}\right)$. Hence by Theorem 5 , we obtain $\mu(\epsilon)=$ $O\left(\epsilon^{2 d-2}\right)$. Let us note that the convergence rates imply $u_{0}(x)=0$ if $\left|p_{0}(x)\right|=\beta$ by Theorem 4. Moreover, we have $u_{0}=\tilde{u}_{\alpha}=0$ on $\left\{x \in \Omega:\left|p_{0}(x)\right| \leq \beta\right\}$ by (3) and (10). This implies $u_{0}=\tilde{u}_{\alpha}=0$ on the set $\left\{x \in \Omega:\left|p_{0}(x)\right| \leq \beta\right\}=\Omega \backslash A$, cf. (10). Using the convergence rate $\left\|p_{0}-p_{\alpha}\right\|_{L^{\infty}(\Omega)}=O\left(\alpha^{d}\right)$ and Theorem 6 , we find

$$
\begin{aligned}
\alpha^{-1}\left|\left(p_{0}-p_{\alpha}, \tilde{u}_{\alpha}-u_{0}\right)\right| & =O\left(\alpha^{d-1}\right)\left\|\tilde{u}_{\alpha}-u_{0}\right\|_{L^{1}(\Omega)} \\
& =O\left(\alpha^{d-1}\right)\left\|\tilde{u}_{\alpha}-u_{0}\right\|_{L^{1}(A)} \\
& \leq O\left(\alpha^{d-1}\right) \mu(M \alpha) .
\end{aligned}
$$

Since by the above considerations we already got $\mu(\epsilon)=O\left(\epsilon^{2 d-2}\right)$ this gives

$$
\left\|u_{0}-\tilde{u}_{\alpha}\right\|_{L^{2}(\Omega)}^{2}=O\left(\alpha^{2 d-1}\right)+O\left(\alpha^{3(d-1)}\right) .
$$

Repeating this process $k$ times until $k(d-1) \geq 2 d-1$ yields

$$
\left\|u_{0}-\tilde{u}_{\alpha}\right\|_{L^{2}(\Omega)}^{2}=O\left(\alpha^{2 d-1}\right),
$$

which finishes the proof.

Together with Theorem 4, this result shows that the requirements of Theorem 3 for convergence rates $d>1$ are sharp. It is an open question, whether the requirement (5) on the active set is also necessary for convergence rates $d \leq 1$. In our opinion, this condition is too strong and has to be relaxed in order to obtain a characterization for convergence rates $d \leq 1$.

\subsection{Necessary Conditions for Exact Reconstruction with $\alpha>0$}

Let us now investigate the case of exact reconstruction. That is, the solutions of the regularized problem $u_{\alpha}$ coincide with the (minimal $L^{2}$-norm) solution $u_{0}$ of the original problem.

Lemma 9. Let us assume that $u_{\alpha^{*}}=u_{0}$ a.e. on $\Omega$ for some $\alpha^{*}>0$. Then $u_{\alpha}=u_{0}$ a.e. on $\Omega$ for all $\alpha \in\left(0, \alpha^{*}\right)$.

Proof. The claim follows from known monotonicity results: The mapping $\alpha \mapsto$ $\left\|u_{\alpha}\right\|_{L^{2}}$ is monotonically decreasing, while $\alpha \mapsto \frac{1}{2}\left\|y_{\alpha}-y_{d}\right\|_{Y}^{2}+\beta\left\|u_{\alpha}\right\|_{L^{1}}$ is monotonically increasing from $(0,+\infty)$ to $\mathbb{R}$, see e.g. [8, Lemma 2.8]. 
Theorem 10. Let us assume that there is $\sigma>0$ such that $u_{a} \leq-\sigma<0<\sigma \leq$ $u_{b}$ a.e. on $\Omega$. Then the exact recovery $u_{\alpha^{*}}=u_{0}$ a.e. on $\Omega$ for some $\alpha^{*}>0$ is equivalent to

$$
\left.\begin{array}{rl}
u_{0} & =0 \quad \text { on }\left\{x \in \Omega:\left|p_{0}(x)\right|=\beta\right\} \quad \text { and } \\
\mu(\epsilon) & =\left|\left\{x \in \Omega: 0<\left|p_{0}(x)\right|-\beta<\epsilon\right\}\right|=0
\end{array}\right\}
$$

for some $\epsilon>0$.

Proof. Let us assume $u_{\alpha^{*}}=u_{0}$ for some $\alpha^{*}>0$. Theorem 9 and Theorem 4 imply $u_{0}(x)=0$ for $x \in\left\{x \in \Omega:\left|p_{0}(x)\right|=\beta\right\}$. Moreover, due to $p_{0}=p_{\alpha^{*}}$ we infer $u_{0}=u_{\alpha^{*}}=\tilde{u}_{\alpha^{*}}$ from Theorem 7 , where $\tilde{u}_{\alpha^{*}}$ is defined by (10). Hence, Theorem 6 implies $\mu\left(\sigma \alpha^{*} / 2\right)=0$.

To prove the converse, let (11) be satisfied for some $\epsilon>0$. Using (6) we obtain

$$
\alpha\left\|u_{0}-u_{\alpha}\right\|_{L^{2}(\Omega)}^{2} \leq \alpha\left(u_{0}, u_{0}-u_{\alpha}\right)=\alpha\left(\chi_{A} u_{0}, u_{0}-u_{\alpha}\right) \leq C \alpha\left|A_{\alpha}\right|,
$$

where $A=\left\{x \in \Omega:\left|p_{0}(x)\right|>\beta\right\}$ and $A_{\alpha}=\left\{x \in A: u_{0}(x) \neq u_{\alpha}(x)\right\}$. Arguing similarly as in [8, Corollary 3.13], we have $\left|A_{\alpha}\right|=0$, and hence $\left\|u_{0}-u_{\alpha}\right\|_{L^{2}(A)}=0$ holds for $\alpha>0$ small enough.

In many applications, the adjoint state $p_{0}$ belongs to $C(\Omega)$. In this case, the result of Theorem 10 shows that an exact reconstruction is only possible if $\left|p_{0}(x)\right| \neq \beta$ for all $x \in \Omega$. This in turn implies either $u_{0} \equiv u_{a}$ or $u_{0} \equiv 0$ or $u_{0} \equiv u_{b}$ on every connected component of $\Omega$.

\section{References}

1. Casas, E., Herzog, R., Wachsmuth, G.: Optimality conditions and error analysis of semilinear elliptic control problems with $\mathrm{L}^{1}$ cost functional. SIAM J. Optim. (to appear, 2012)

2. Deckelnick, K., Hinze, M.: A note on the approximation of elliptic control problems with bang-bang controls. Comput. Optim. Appl. 51(2), 931-939 (2012)

3. Engl, H.W., Hanke, M., Neubauer, A.: Regularization of inverse problems. Mathematics and its Applications, vol. 375. Kluwer Academic Publishers Group, Dordrecht (1996)

4. Felgenhauer, U.: On stability of bang-bang type controls. SIAM J. Control Optim. 41(6), 1843-1867 (2003)

5. Neubauer, A.: Tikhonov-regularization of ill-posed linear operator equations on closed convex sets. J. Approx. Theory 53(3), 304-320 (1988)

6. Tröltzsch, F.: Optimal Control of Partial Differential Equations. Graduate Studies in Mathematics, vol. 112. American Mathematical Society, Providence (2010); Theory, methods and applications, Translated from the 2005 German original by J. Sprekels

7. Wachsmuth, D., Wachsmuth, G.: Convergence and regularization results for optimal control problems with sparsity functional. ESAIM Control Optim. Calc. Var. 17(3), 858-886 (2011)

8. Wachsmuth, D., Wachsmuth, G.: On the regularization of optimization problems with inequality constraints. Control and Cybernetics (2011) (to appear) 